\title{
Two-dimensional gel-based protein standardization verified by western blot analysis
}

Hisao Haniu, Daisuke Watanabe, Yusuke Kawashima, and Hiroyuki Matsumoto

\begin{abstract}
In data presentation of biochemical investigation the amount of a target protein is shown in the $y$-axis against the $x$-axis representing time, concentrations of various agents, or other parameters. Western blot is a versatile and convenient tool in such analysis to quantify and display the amount of proteins. In western blot, so-called "housekeeping" gene product(s), or "housekeeping proteins", are widely used as internal standards. The rationale of using housekeeping proteins for standardization of western blot is based on the assumption that the expression of chosen housekeeping gene is always constant, which could be false under certain physiological or pathological conditions. We have devised a two-dimensional gelbased standardization method in which the total proteins of each sample are determined by scanning the total protein density of two-dimensional gels and the expression of each protein is quantified as the density ratio of each protein divided by the density of the total proteins on the two-dimensional gel. The advantage of this standardization method is that it is not based on any presumed "housekeeping proteins" that are supposed to be being expressed constantly under all physiological conditions. We will show that the total density of a two-dimensional gel can render a
\end{abstract}


reliable protein standardization parameter by running western blot analysis on one of the proteins analyzed by two-dimensional gels.

Key words: protein standardization; total protein density of a two-dimensional gel; ratio of a protein against the total gel density; quantitative western blot

Running Title: Standardization Based on Total Protein Density

Affiliation:

HISAO HANIU

Shinshu University, Matsumoto, Nagano, Japan

DAISUKE WATANABE

University of Oklahoma Health Sciences Center, Oklahoma City, OK, USA

Yusuke Kawashima

University of Oklahoma Health Sciences Center, Oklahoma City, OK, USA

HiROYUKi MATSUMoto

University of Oklahoma Health Sciences Center, Oklahoma City, OK, USA 


\section{Introduction}

Western blot analysis of proteins existing in biological specimens is one of the most frequently used experimental protocols in biochemistry and molecular biology. Results will be interpreted based on the amount of expressed protein on the y-axis against variables on the $\mathrm{x}$-axis. There has been always a concern in western blot analysis with regard to how to standardize the measurement. Traditionally, so called "housekeeping proteins" that are presumably expressed constantly are used for internal standards. Recently, however, the notion that expression of housekeeping proteins is constant is challenged $[1,2]$. We have devised a method to quantitate multiple proteins displayed by two-dimensional gel electrophoresis [3]. After scanning the gels we quantified each protein standardized against the total protein density of each gel. By utilizing this protein quantification method we showed that proteins expressed in a neonatal mouse retina can be mapped along the developmental time axis and that we can classify proteins according to the expression profile (proteomic trajectory mapping). The standardization principle used in this technique can be applied to western blot on one-dimensional gel electrophoresis. In this paper we will briefly explain the protein stain-based quantitation performed on two-dimensional gel electrophoresis, which we designate "2-DE Density Method", and the correspondence of the result of 2-DE Density Method to that of one-dimensional gel-based quantitative western blot designated "Western Blot Method." 


\section{Materials}

1. Two-dimensional gel electrophoresis (2DE) apparatus for a glass tube-based IEF system followed by SDS-PAGE. The apparatus was described previously in a chapter of this protocol series.

2. Developing retinas from neonatal C57BL/6 mice were dissected at postnatal (P) day at $1,3,5,7,9,14,21$, and adult (>28 days). Retinas were flash-frozen in liquid nitrogen and stored at $-80^{\circ} \mathrm{C}$ until use.

3. IEF lysis solution containing $9 \mathrm{M}$ urea, 4\% CHAPS, 2\% Bio-Lyte pH 3-10 (BioRad, Hercules, CA), 40 mM Tris, and 5\% b-mercaptoethanol.

4. A transmission scanner (PowerLook III; UMAX Technologies, Dallas, TX) at 14-bit depth and a resolution of $300 \mathrm{dpi}$.

5. Kodak Photographic Step Tablet No. 2 (Catalog Number 152 3398) for calibration of the scanner.

6. Progenesis Workstation software (Nonlinear Dynamics, Newcastle upon Tyne, UK).

7. Li-Cor Odyssey Infrared Gel Scanner.

8. Rabbit-anti-mouse IgG against Nrdg-1 (Santa Cruz sc-19464, polyclonal goat $\operatorname{IgG})$.

9. IR fluorescent dye conjugated with donkey IgG; Li-Cor IRDye ${ }^{\circledR} 800 \mathrm{CW}$ antigoat IgG (H+L) P/N 926-32214.

10. BCA ${ }^{\mathrm{TM}}$ Protein Assay Kit (Pierce, Rockford, IL).

11. SDS-PAGE apparatus; NuPAGE® Novex Midi Gel System (Invitrogen, Carlsbad, CA). 
12. Electroblotting apparatus; iBlot ${ }^{\mathrm{TM}}$ Invitrogen, Carlsbad, CA).

\section{Methods}

1. Two-dimensional gel electrophoresis (2DE) was conducted by a glass tubebased IEF system followed by SDS-PAGE. The protocol was described previously in a chapter of this protocol series [4].

2. Developing retinas from neonatal C57BL/6 mice were dissected at postnatal (P) day at $1,3,5,7,9,14,21$, and adult ( $>28$ days). Six to 16 retinas harvested at each developmental stage were homogenized with a lysis solution containing 9 M urea, 4\% CHAPS, 2\% Bio-Lyte pH 3-10 (BioRad, Hercules, CA), $40 \mathrm{mM}$ Tris, and $5 \% \mathrm{~b}$-mercaptoethanol in a water bath sonicator at $10^{\circ} \mathrm{C}$. After centrifugation at $16000 \mathrm{xg}$ for $15 \mathrm{~min}$ at $4^{\circ} \mathrm{C}$, the supernatants were collected and the protein contents were assayed by Coomassie Plus protein assay (BioRad) and stored at $-85^{\circ} \mathrm{C}$ until use.

3. One set of 2-DE analysis consists of total proteins extracted from P1, P3, P5, P7, P9, P14, P21, and adult retinas. Since the volume of retinal tissue increases as it matures, the number of retinas at each developmental stage was adjusted accordingly; the number of retinas varied from sixteen to six. 2-DE of each retinal sample containing 400 microgram proteins was performed four times $(n=4)$. After focusing, IEF gels were equilibrated and applied to the second dimension on 11\% SDS-PAGE. The 2-DE gels were stained by CBB R-250 and destained. The gels were sandwiched in between 
cellophane membranes and air-dried. Four sets of 2-DE analyses $(n=4)$ were conducted in order to statistically evaluate the numeric data.

4. 2-DE Density Method: Each 2-D gel was stained with CBB and scanned by a transmission scanner (PowerLook III; UMAX Technologies, Dallas, TX) at 14bit depth and a resolution of $300 \mathrm{dpi}$. The scanner was calibrated up to the transmission OD of 3.1 using a Kodak Photographic Step Tablet No. 2

(Catalog Number 152 3398), ensuring linearity. The scanned gel images were analyzed by Progenesis Workstation software (Nonlinear Dynamics, Newcastle upon Tyne, UK) to quantify the protein spots. Figure 1 represents a quadruple set of eight 2-DE gels showing the whole image of each gel of total retinal proteins of at P1, P3, P5, P7, P9, P14, P21, and Adult stage, respectively. In this illustration, a part of each 2-DE gel that contains a protein of interest, in this case Ndrg1, is shown. The Progenesis software automatically defines the boundaries of 2-DE gel spots for each gel and also assigns the set of corresponding protein spots on the eight gels. The assignment of the 2-DE gel spot boundaries were visually inspected and corrected, when necessary. The correspondence of each 2-DE gel spot between eight gels was also inspected visually and correction was made, when necessary. The percentage of each protein spot after the normalization was expressed as percentile (\%).

5. We conducted cluster analysis of these retinal proteins and grouped them into four groups, i.e., Juvenile-type, Transient-type, Adult-type, and Constanttype, depending on their expression patterns [3]. 
6. Western Blot Method: Using a separate batch of developing C57BL/6 retinas, SDS-PAGE was run in order to evaluate the protein expression profile of one of the Transient-type proteins Ndrg1, which was shown to be expressed transiently higher around stages P5 P14. For each developmental stage, 10 micrograms of total retinal proteins were run on an SDS-PAGE by using NuPAGE® Novex Midi Gel System according to the manufacturer's instruction.

7. The gels were transferred electronically onto a PVDF membrane by using an electroblotting apparatus (Invitrogen iBlot ${ }^{\mathrm{TM}}$ ) according to the manufacturer's instruction. The background proteins were blocked by Odyssey Blocking Buffer (Li-CoR) solution. The PVDF membrane was incubated with the goat primary antibody against mouse Ndrg1 and washed twice. The PVDF membrane was incubated with the secondary donkey antibody against goat IgG tagged with IR dye, Li-Cor IRDye ${ }^{\circledR} 800 \mathrm{CW}$ antigoat IgG $(\mathrm{H}+\mathrm{L}) \mathrm{P} / \mathrm{N}$ 926-32214, and washed three times. The gel image was scanned with Li-CoR IR scanner and quantified. The overall protocol for the quantitative western blot is illustrated in Figure 2.

8. In Figure 3 the developmental expression profiles of Ndrg1 obtained from two independent methods, i.e., the 2-DE Density Method and the Western Blot Method, are shown. The results indicate that the 2-DE Density Method and the Western Blot Method project a consistent protein expression profile during the development of neonatal retina, assuring the reliability of using total protein density for standardization. 


\section{Notes}

1. The data shown in Figure 3 illustrates; 1 ) that the total protein density on a 2-DE can be a reliable measure for standardization of a protein expressed in the system, and 2) that quantitative western blot using an infrared fluorescent dye-tagged second antibody to detect the primary antibody bound with the antigen protein separated on SDS-PAGE would be a simpler protocol to quantitate a protein compared to that by the 2-DE Density Method.

2. It should be noted that the 2-DE Density Method is required in order to profile the two-dimensional protein map with each protein identified by peptide mass fingerprinting [3]. After the protein map is established, however, the Western Blot Method will serve more efficient and less time consuming protocol for the quantification of protein in a set of biological samples.

\section{Acknowledgment}

The projects described in this article were partially supported by Oklahoma Center for Advancement of Science and Technology (OCAST) Health Research Program Grant HR10-120 and NIH R21 EY017888. 


\section{References}

1. Li R, Shen Y: An old method facing a new challenge: re-visiting housekeeping proteins as internal reference control for neuroscience research. Life sciences 2013, 92(13):747-751.

2. Kim HJ, Na JI, Min BW, Na JY, Lee KH, Lee JH, Lee YJ, Kim HS, Park JT:

Evaluation of Protein Expression in Housekeeping Genes across

Multiple Tissues in Rats. Korean journal of pathology 2014, 48(3):193-200.

3. Haniu H, Komori N, Takemori N, Singh A, Ash JD, Matsumoto H: Proteomic trajectory mapping of biological transformation: Application to developmental mouse retina. Proteomics 2006, 6(11):3251-3261.

4. Matsumoto H, Haniu H, Kurien BT, Komori N: Two-dimensional gel electrophoresis: glass tube-based IEF followed by SDS-PAGE. Methods in molecular biology 2012, 869:267-273.

\section{Figure Legends}

Figure 1. Two-dimensional gel profiles of mouse retinal proteins during neonatal development P1 through adult. The gels were stained with CBB R-250. The region of each gel representing $\mathrm{Ndrg} 1$ ( $\mathrm{N}$-myc downstream regulated gene 1; $45 \mathrm{kDa}, \mathrm{pI}=5.7$ ) is shown. A thicker border among other borders illustrates the Ndrg1 protein. For each developmental stage, four independent mouse groups $(n=4)$ were analyzed. The protein expression kinetics (proteomic trajectory map) is illustrated in Fig. 2, 
“2-DE Density Method”. These portions of 2-DE gel images were automatically extracted from the original 2-DE gels by Progenesis software.

Figure 2. Illustrated workflows for quantitative western blot using infrared fluorescent-labeled secondary antibody detected by Odyssey Infrared Imaging System (Li-Cor). After electroblotting proteins separated on SDS-PAGE onto a PVDF membrane the proteins was visualized by a primary antibody specific to the protein of interest (e.g., goat anti-Ndrg1 antibody in this case), followed by the secondary donkey antibody against goat IgG tagged with IR dye, Li-Cor IRDye® ${ }^{\circledR} 800 \mathrm{CW}$. The PVDF membrane was scanned by Odyssey Infrared Scanner (Li-Cor) and the software of the Odyssey scanner analyzed the images (http://www.licor.com/bio/applications/quantitative western blots/).

Figure 3. "(2-DE) Density Method" and "Western Blot (Method)" characterize the proteomic trajectory map of the target protein Ndrg1 consistently. The error bars indicate standard deviations. 


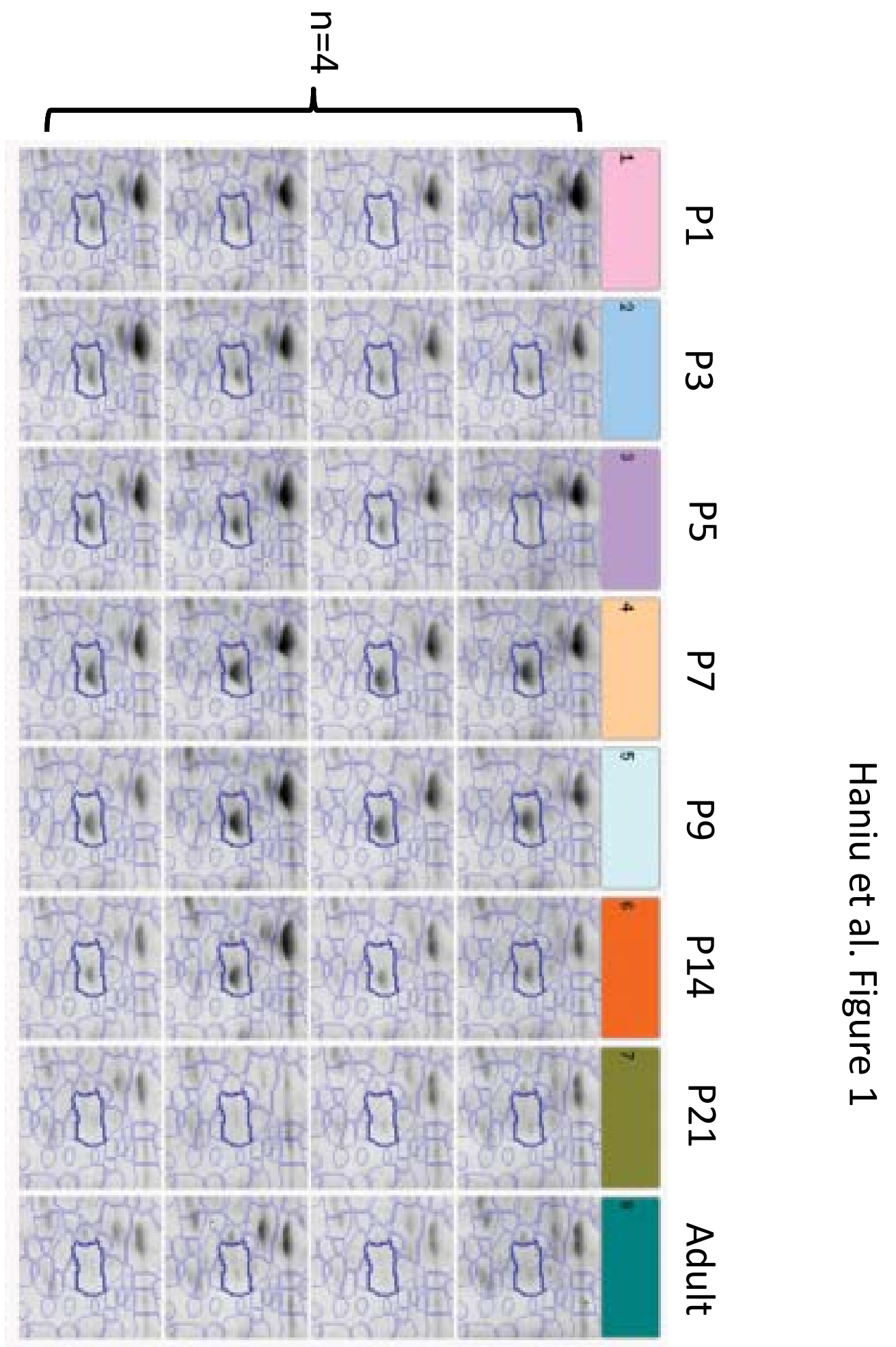


Haniu et al. Figure 2
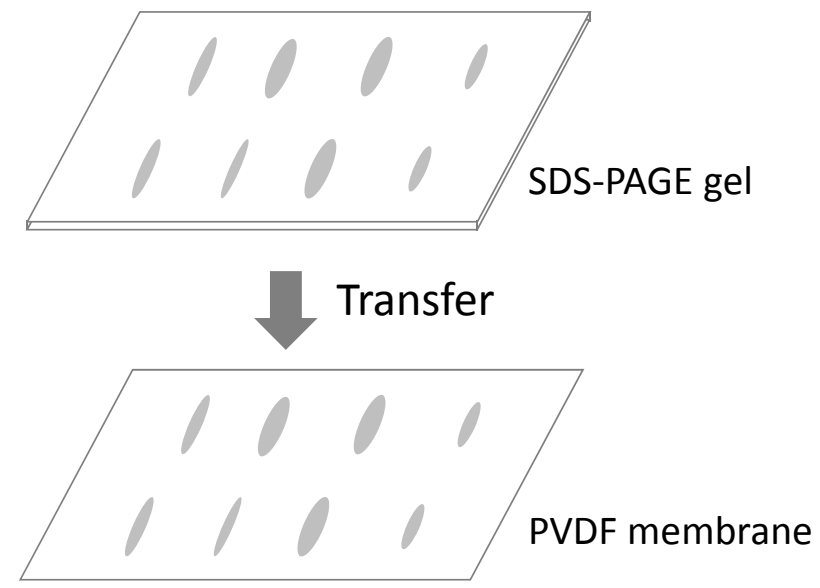

Primary antibody

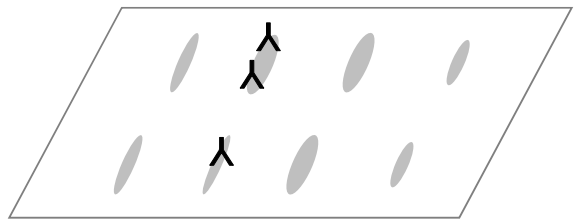

Secondary antibody tagged with IR dye

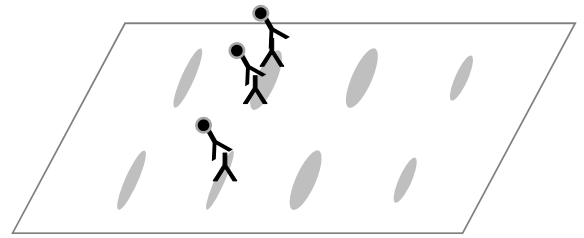

IR fluorescent Detection
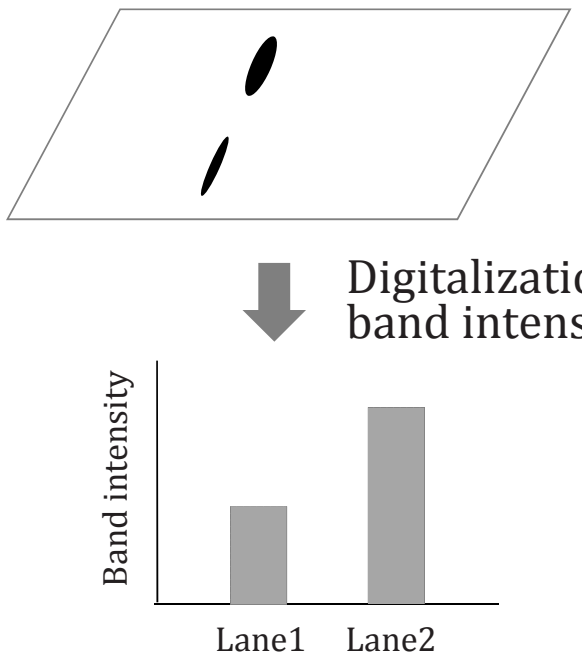


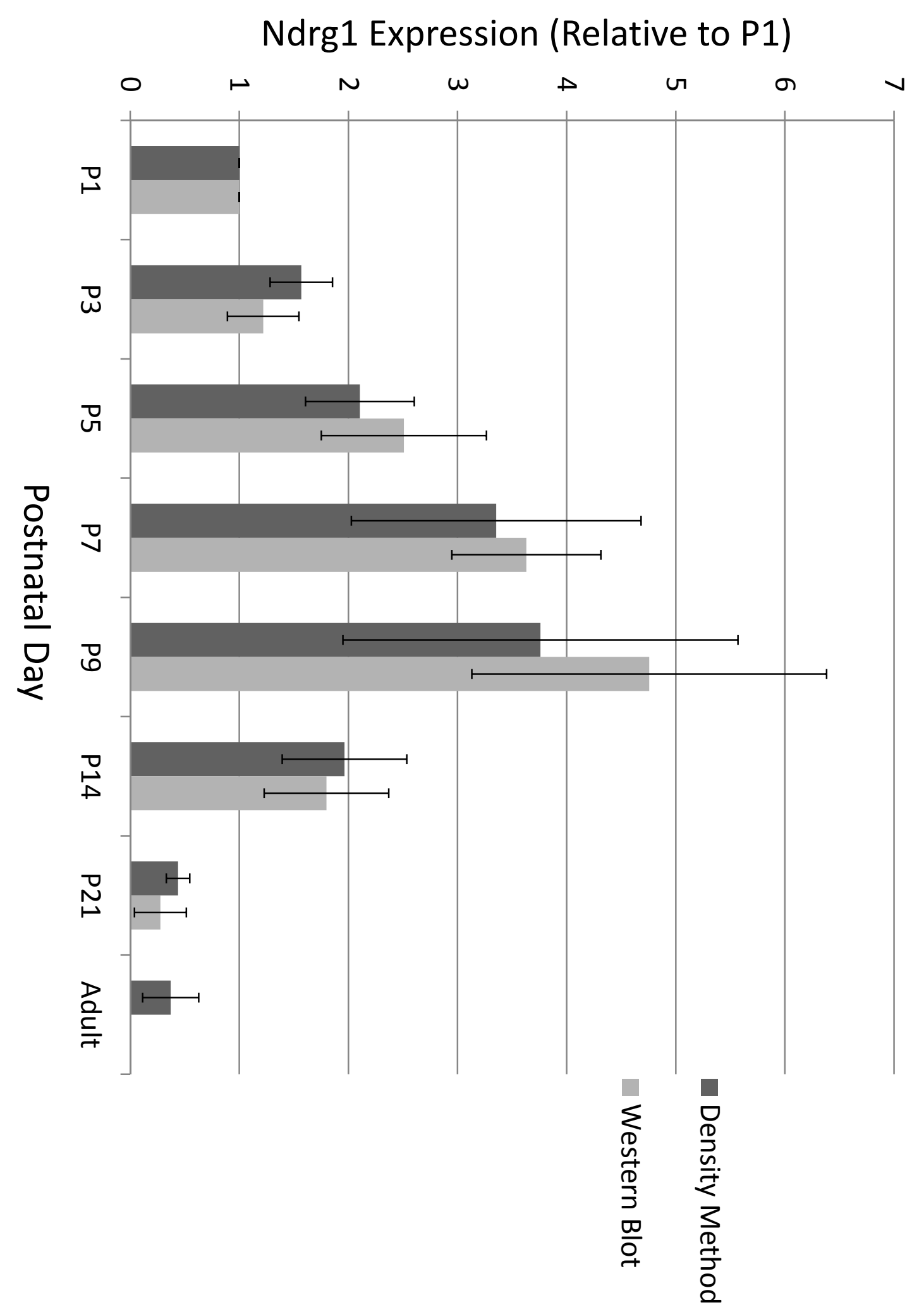

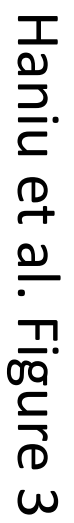

